\title{
Genetic Engineering and Animal Infectious Diseases
}

\author{
Giovanni Di Guardo* \\ University of Teramo, Faculty of Veterinary Medicine, Piazza Aldo Moro, 45 - 64100 Teramo, Italy
}

Received: December 23, 2013; Accepted: December 27, 2013; Published: December 28, 2013

*Corresponding author: Giovanni Di Guardo, University of Teramo, Faculty of Veterinary Medicine, Italy, Tel: +39-861-266933; Email: gdiguardo@ unite.it

\begin{abstract}
The present Letter to the Editor deals with the potential risks related to genetic engineering/selection in favour of resistance to infectious diseases in animals. Indeed, this appears to be a valuable option to counteract the serious risk of extinction faced by $15-40 \%$ of living species worldwide by the year 2050 .
\end{abstract}

Genetic engineering could represent a crucial strategy in facing the risk of extinction for $15-40 \%$ of living species by the year 2050 [5]. Genetic engineering might also affect the epidemiology of infectious diseases among wild and domestic animals, with special reference to host-parasite interaction, thereby linking its effects with those of climate change and human activities. Indeed, genetic manipulation in favor of resistance to infection may be a strategic option, as clearly exemplified by the positive results obtained in the ovine population of the European Union after more than a decade of genetic selection for resistance to sheep scrapie, the animal and human prion disease "prototype" [2]. Nevertheless, when applying genetic engineering/selection for resistance to infectious diseases, special emphasis should be also placed on the possibility that one or more "undesidered" genetic traits could become phenotypically expressed and vertically transmitted within the concerned animal species and populations. An illuminating example in this direction is represented by the devastating morbilliviral epidemics occurred in past and recent years among wild terrestrial carnivores [3] and free-living pinnipeds and cetaceans [1]. Because many from the expanding range of Morbillivirus-susceptible hosts are top-predators, they may accumulate high tissue loads of immunotoxic and endocrinedysrupting pollutants, such as organochlorines (OCs). In this respect, a "side-effect" of genetic manipulation for resistance to morbilliviral infection could lie in an enhanced expression level by target cells of estrogen receptors and/or aryl hydrocarbon receptors, which are known to specifically bind both OCs and other toxic environmental contaminants [4], with a potentially increased toxicity of these compounds for the aforementioned hosts.

On the basis of what above, in-depth studies on this challenging issue are strongly needed.

\section{References}

1. Di Guardo G (2012) Morbillivirus-host interaction: lessons from aquatic mammals. Frontiers in Microbiology 3: 431.

2. Gryspeirt A, Gubbins S (2013) A Bayesian framework to assess the potential for controlling classical scrapie in sheep flocks using a live diagnostic test. Epidemics 5: 123-130.

3. Roelke-Parker ME, Munson L, Packer C, Kock R, Cleaveland S, et al (1996) A canine distemper virus epidemic in Serengeti lions (Panthera leo). Nature 379: 441-445.

4. Shanle EK, Xu W (2011) Endocrine disrupting chemicals targeting estrogen receptors: signaling, identification and mechanisms of action. Chemical Research in Toxicology 24: 6-19.

5. Thomas MA, Roemer GW, Donlan CJ, Dickson BG, Matocq M, et al (2013) Ecology: Gene tweaking for conservation. Nature 501: 485486 . 